\title{
Novel Dual-FCL Connection with Neuro-fuzzy controller for Adding DG to a Power Distribution Utility
}

\author{
P. Vasudevarao ${ }^{1}$, Raghavaiah.Katuri ${ }^{2}$ \\ ${ }^{I}$ (PG Scholar, Department of Electrical and Electronics Engineering, QISCET, Ongole.) \\ ${ }^{2}$ (Assistant Professor, Department of Electrical and Electronics Engineering, QISCET, Ongle.)
}

\begin{abstract}
The increasing capacity of power systems, larger available fault currents may cause the existing circuit breakers to become under-rated. Excessive dynamic stress during severe faults may result in failure of circuit breakers to open. Larger fault currents may also adversely influence synchronization of the generators, shorten the life of electric machines, or even cause complete failure of equipment. Fault current limiters (FCLs) are able to prevent all of these disruptive events by limiting currents during faults and by increasing the robustness of generator synchronization. The DUAL FCL placement technique is demonstrated in a power network containing four buses in a ring arrangement. The system receives power from turbo-generators (36 $M W$ ). This dual-FCL connection reduces fault current contributions of the DG by 98.5\% (from 41 to $1 \mathrm{kA}$ ), and maintains the circuit breakers within their duty limits when a three-phase fault occurs at a bus connected to the $D G$. Otherwise, when a three-phase fault occurs in the infinite system, this connection limits the fault current contribution of the DG from 4.8 to $0.4 \mathrm{kA}$, and frequency oscillations of the $D G$ from 0.14 to $0.01 \mathrm{~Hz}$. The power continuity in the local network is improved; as the voltage sag at the bus connected to the DG is reduced by $98 \%$ during and after serious short-circuit faults. In this in order to control effectively Neuro-Fuzzy controller is used. And the simulation is done using Matlab/Simulation and simulation results are examined in different cases.
\end{abstract}

Keywords: About DG, dual-FCL, HTS, Neuro-fuzzy.

\section{Introduction}

Fault current limiters (FCLs) offer a variety of benefits for existing and future electrical distribution systems. These include sub-cycle operation in response to faults [1], [2], reduced damage at the point of fault [3], [4], and the opportunity for increased network interconnection [5]. Fault current limiting devices are becoming increasingly important because of the connection of distributed generation (DG) [6] and in systems where fault levels are inherently high, such as marine vessels [3], [7]. There is a desire for power networks to improve in reliability and efficiency, which may necessitate increased interconnection, which generally increases fault levels [8]. However, the coordination of protection can be difficult, expensive, or impractical on distribution networks with DG [9] and on networks which are highly-interconnected [10] because coordination often requires protection signalling. This paper analyzes the Neuro-fuzzy logic based control of Dual FCL, as well as offering the typical benefits to power system performance from fault current limitation.. Section II describes the FCL and power system models, and Section III illustrates typical Neuro_fuzzy Controller. IV.A novel dual FCL-connection. Section V indicated the Simulation results in different cases

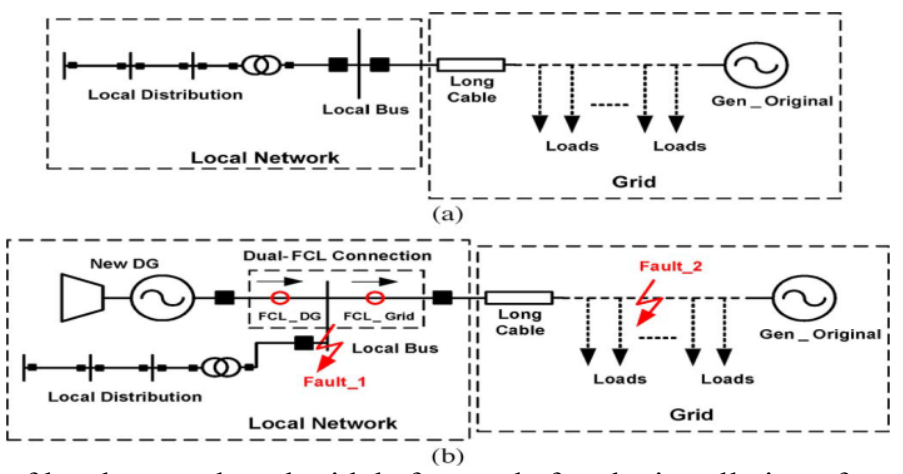

Fig. 1. Connections of local network and grid, before and after the installation of new DG and dual-FCL connection 


\section{Fault Current Limiters}

Before technologies can be considered for the application of limiting a distributed generator's fault current contribution, the operating conditions and requirements of such a limiter must first be established. The existing technologies can then be evaluated for their suitability for such an application by ensuring that any proposed device meets the requirements. The first requirement for the FCL is that it must operate at the distribution voltage level. According to a utility survey in [13] in which utilities were asked to describe their present distribution systems and predicted future system, utilities responded that the most prevalent voltage class is at $15 \mathrm{kV}$. A typical radial distribution system is shown in Figure 2.

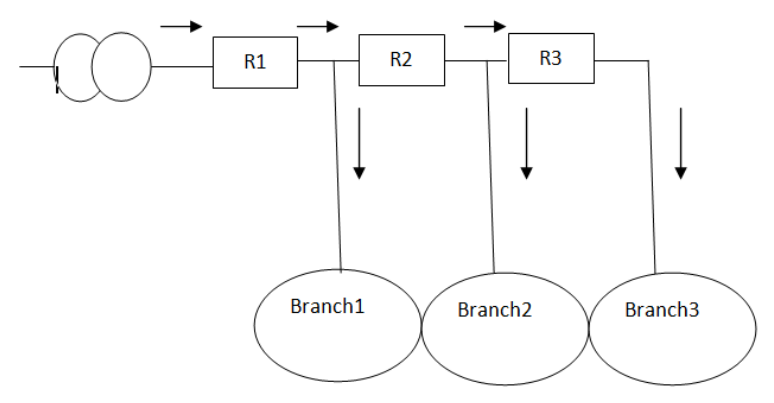

Fig 2: Radial distribution with Relay protection

The distribution system shown in Figure 3 is an example in which the addition of DG to the system in Figure 2 would impact the existing relay coordination. If a fault were to occur in Branch 1 or Branch 3, the DG would likely contribute to the fault current, with the current flowing from the DG back onto the main feeder, towards the fault. The current that flows through relay R2 would then be different than if the DG had not been added, so that the coordination between the 3 relays, R1, R2, and R3, would be affected.

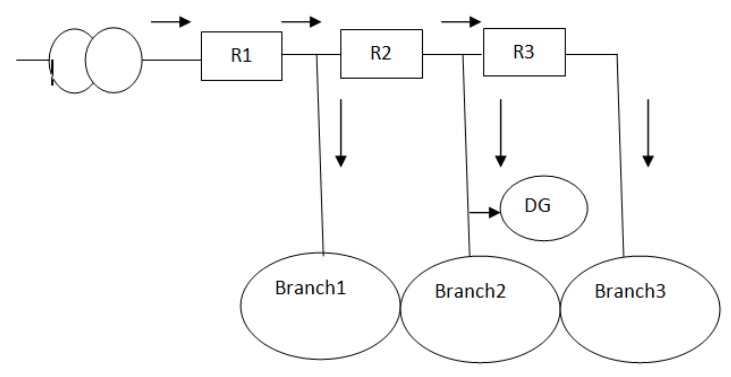

Fig3: Radial distribution with DG

To prevent the DG from supplying fault current onto the main feeder, the FCL should be placed between the DG and the main feeder, along the distribution line off of the main feeder leading to the DG and Branch 2 as shown by the $\mathbf{X}$ in Figure 3. The fault current limiter's operation must follow specific guidelines in such a placement. When there is no fault in the system, the FCL must not affect the system. If a fault occurs in the system outside of Branch 2, the FCL must limit the current that will flow from the DG to the fault. In the last mode of operation, for faults inside Branch 2, the FCL must not operate, preserving the fault current seen by the relay, R2. To be able to operate in the manner described, limiting the fault current in one direction while having no effect on the other, the fault current limiter needs the ability to be selectively turned on and off based on the direction of the sensed current. Finally, for the FCL to be viable, it will need to introduce almost no losses during the steady-state operation of the system, and be able to sustain repeated operations with low maintenance.

\section{A. Passive Limiters:}

Traditionally, fault current limiters implemented in practice and researched have been of the passive type: devices that are permanently connected to the power system and do not need to be"turned on" or controlled by an external signal. When a fault occurs, the nature of these devices is such that the over current is automatically reduced or limited. Passive devices include series inductors [14], and superconducting fault current limiters [15], [16]. While series inductors are inexpensive and require low maintenance, they cause a voltage drop during steady state operation and have poor limiting performance compared to newer FCL 
topologies. fault current limiters have no resistive or ohmic losses during steady state operation, and can effectively limit a fault current, but uncertainties regarding cooling losses, regular maintenance, and the ongoing research to develop more economically cooled high temperature superconductor devices reduce the viability of such devices.

\section{B. Solid State Limiters:}

With advances in switch technology that have made them suitable for the voltage and power levels necessary for power applications, power electronic switches can be used to build devices that could sustain repeated operations with high reliability and without wearing out [17]. There are 2 main types of solid state current: resonance based devices and impedance switch-in limiters. Resonance based limiters include devices proposed in [18]-[20]. The basis for their operation is that because power is transmitted at a fixed AC sinusoidal frequency, the impedance of a LC-resonant circuit can be tuned such that the impedance of the device during steady state operation is nearly zero. During a fault, power electronic switches isolate a capacitor or inductor from the device, introducing large impedance into the system. The limitations of resonance based limiters are that they can because voltage sags during faults, current limitation effectiveness declines as distance from substation increases, large infrastructure for capacitors is required, and tuning of devices is necessary to ensure low impedance. The basis for their operation is that impedance is placed in series with the distribution line. A pair of Gate-Turn-Off (GTO) thyristor switches are placed in shunt with this impedance, and operated during alternate half cycles of the voltage waveform to present a low impedance path. In the event of a fault, the gating signals to the GTO switches are blocked, resulting in large impedance being introduced into the system, limiting the current. However, these limiters introduce switching losses as the power flows through the power electronic switches during steady state operation and the long term reliability of these devices is questionable because of the continuous switching. Recently, in 2004, Meyer, Koellensperger, and De Doncker proposed several new topologies of switched current limiters in [24], but came to the conclusion that the high operation expenses for losses made all other costs (i.e. initial cost, maintenance) negligible.

\section{Solid-State Switch/Mechanical Switch Hybrid Current Limiter:}

While solid-state fault current limiters offer many advantages to the passive limiters, the switching losses are a major drawback. Mechanical breakers and switches exist that have virtually no closed circuit losses, but they cannot operate quickly and so are unable to commutate the voltage across their contacts. A novel idea is to use a power electronic switch path as a commutation aid for a mechanical switch to open. With a power electronic switch path providing an alternate low impedance current path, the mechanical switch should be able to open without the arcing problems typically associated with mechanical switches opening at high voltage. In [25], Steurer et al. proposes such a hybrid fault current limiter (and circuit breaker) based around a new fast mechanical switch designed by the same authors. The device is capable of operating in a system with a single phase voltage of $12 \mathrm{kV} \mathrm{rms}$ and a steady state current of $1 \mathrm{kA}$.

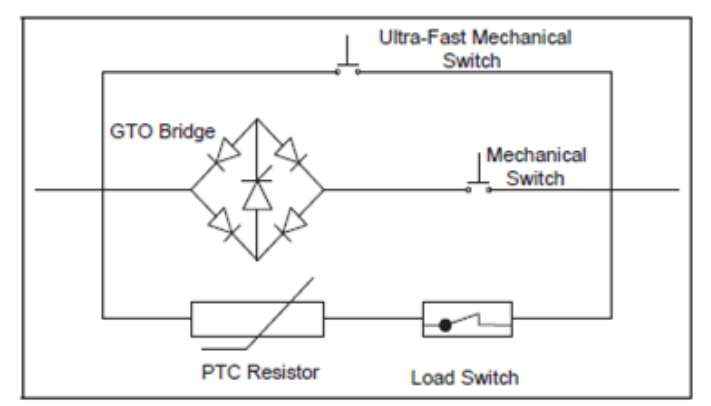

Fig 4: Novel Hybrid Fault Current Limiter

During the steady state operation of the system, all three mechanical switches are closed and the GTO in the bridge is gated on. When a fault occurs, the Ultra-Fast Mechanical Switch opens within several hundred microseconds [25]. After the complete commutation of the current to the GTO Bridge, the GTO is gated off, forcing the current through the positive temperature coefficient (PTC) resistor, whose resistance increases as its temperature rises. The mechanical switch in series with the GTO Bridge disconnects shortly thereafter to prevent a high voltage across the GTO Bridge. The load switch interrupts the limited current for circuit breaker operation. This hybrid fault current limiter meets the original application requirements $n$, the circuit breaker operation of the proposed device is not needed in use in the application described in this paper, so the fault current limiter in Figure 4 can be modified to omit the load switch. Additionally, for the simulations, the PTC 
resistor in the device is replaced by a fixed value resistor, a feasible Implementation given the energy dissipation capabilities of high voltage resistors currently available.

\section{Neuro-Fuzzy Controller}

We consider a multi-input, single-output dynamic system whose states at any instant can be defined by " $\mathrm{n}$ " variables X1, X2,..,Xn. The control action that derives the system to a desired state can be described by a well known concept of "if-then" rules, where input variables are first transformed into their respective linguistic variables, also called Fuzzification. Then, conjunction of these rules, called inferencing process, determines the linguistic value for the output. This linguistic value of the output also called fuzzified output is then converted to a crisp value by using Defuzzification scheme. All rules in this architecture are evaluated in parallel to generate the final output fuzzy set, which is then defuzzified to get the crisp output value. The conjunction of fuzzified inputs is usually done by either min or product operation (we use product operation) and for generating the output max or sum operation is generally used. For Defuzzification, we have used simplified reasoning method, also known as modified centre of area method. For simplicity, triangular fuzzy sets will be used for both input and output. The whole working and analysis of fuzzy controller is dependent on the following constraints on Fuzzification, Defuzzification and the knowledge base of an FLC, which give a linear approximation of most FLC implementations.

Constraint 1: The Fuzzification process uses the triangular membership function

.Constraint 2: The width of a fuzzy set extends to the peak value of each adjacent fuzzy set and vice versa. The sum of the membership values over the interval between two adjacent sets will be one. Therefore, the sum of all membership values over the universe of discourse at any instant for a control variable will always be equal to one. This constraint is commonly referred to as fuzzy partitioning.

Constraint 3: The Defuzzification method used is the modified centre of area method. This method is similar to obtaining a weighted average of all possible output values. An example of a very simple neuro fuzzy controller with just four rules is depicted in Figure 5. This architecture can be readily understood as a "neural-like" architecture. At the same time, it can be easily interpreted as a fuzzy logic controller. The modules X1 and X2 represent the input variables that describe the state of the system to be controlled. These modules deliver crisp input values to the respective membership modules ( $\mu$-modules) which contain definitions of membership Functions and basically fuzzify the input. Now, both the inputs are in the form of linguistic variables and membership associated with the respective linguistic variables. The $\mu$-modules are further connected to Rmodules which represent the rule base of the controller, also known as the knowledge base. Each $\mu$-module gives to its connected R-modules, the membership value $\mu$ (xi) of the input variable Xi associated with that particular linguistic variable or the input fuzzy set. The R-modules use either min-operation or productoperation to generate conjunction of their respective inputs and pass this calculated value forward to one of $v$ modules. The $v$-modules basically represent the output fuzzy sets or store the definition of output linguistic variables. If there are more than two rules affecting one output variable then either their sum or the max is taken and the fuzzy set is either clipped or multiplied by that resultant value. These $v$-modules pass on the changed output fuzzy sets to C-module where the Defuzzification process is used to get the final crisp value of the output.

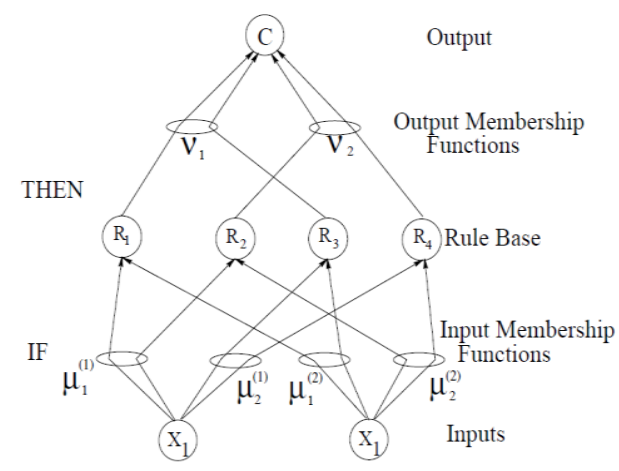

Fig. 5. Architecture of four rule fuzzy controller from neural networks point of view

The architecture given in Figure 5 of a fuzzy logic controller resembles a feed forward neural network. The X-, R-, and C-modules can be viewed as the neurons in a layered neural network and the $\mu$ - and $v$-units as the adaptable weights of the network. The X-module layer can easily be identified as the input layer of a multiinput neural network whereas the $\mathrm{C}$-module layer can be seen as the output layer. The R-module layer serves as the hidden or intermediate layer that constitutes the internal representation of the network. The fact that one $\mu$ - 
module can be connected to more than one R-module is equivalent to the connections in a neural network that share a common weight. This is of key importance for keeping the structural integrity of the fuzzy controller.

\section{Novel Dual Fcl-Connection}

The dual-FCL connection consists of two FCLs (FCL_DG and FCL_Grid). Local Bus connects the new DG, Grid, and the Local Distribution. The local network consists of newDG, Dual-FCL Connection and Local Distribution, while the Grid consists of Long-Cable, Loads, and Gen_Original.

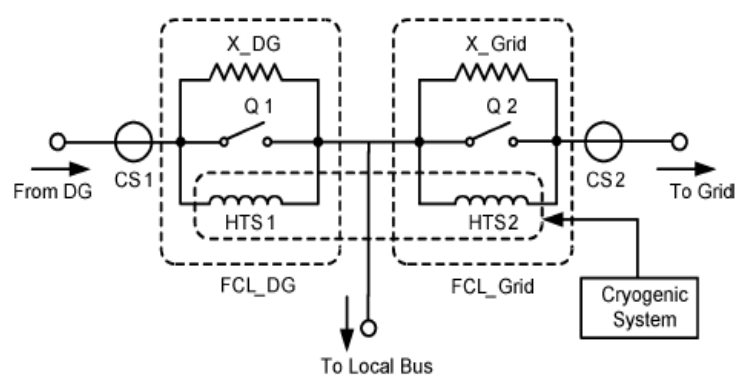

Fig. 6. Topology of dual-FCL connection.

Fault_1 present the faults occurring at the local network, and Fault_ 2 present the faults in the Grid. The local loads can receive power both from the new DG and the Grid. The excessive power from new DG can be fed backward into the Grid.

\section{A. Topology of Dual-FCL Connection:}

To realize the function of this dual-FCL connection, one topology of dual-FCL connection is proposed here, as shown in Fig. 6 FCL_DG consists of X_DG, Q1 and HTS1, while FCL_Grid consists of X_Grid, Q2 and HTS2. X_DG and X_Grid are fault-current-limiting elements; Q1 and Q2 are power switches, like GTO and IGBT, which help: - In the shielding of the FCL function by bypassing its parallel elements during faults HTS1 and HTS2 are two three-phase high-temperature superconducting wires. They share one cryogenic system because of their close locations. In this topology, this equipment can realize the function of two FCLs, but its volume is much smaller than two separate FCLs; CS1 and CS2 are current sensors which are in series with FCLs and communicate in high frequency (several $\mathrm{kHz}$ ). During normal operations, Q1 and Q2 are open, while HTS1 and HTS2 stay in superconducting statuses. Currents flow through these two superconducting wires. Both of FCLs stay at low impedance statuses nearly zero. When a fault occurs at Local Bus (Fault_1), HTS1 and HTS2 turn into non-superconducting statuses due to over current. A signal is sent to Q2 to close thereby bypassing HTS2, while Q1 keeping open. The fault current flows through X_DG and Q2. Under this condition, the dual-FCL connection controller trips FCL_DG, instead of FCL_Grid. The fault current flowing from DG into Local Bus is limited by X_DG to avoid disturbing the original protection relay scheme. The fault current, flowing into Local Bus, remains at the same level as when there is no DG installed. So, the fault can be cleared by the original protection relay scheme. The circuit breakers (CBs) at Local Bus do not need to be upgraded with the new ones of higher interrupting capacity ratings. When a fault occurs at other places in the Grid (Fault_2), HTS1 and HTS2 turn into non-superconducting statuses due to over current. A signal is sent to Q1 to close thereby bypassingHTS1, while Q2 keeping open. The fault current flows through X_Grid and Q1. X_Grid limits fault currents flowing into the Grid. Under this condition, the fault can be cleared by the original protection relay scheme which is not disturbed by fault currents contributed from DG. The local loads at Local Bus are isolated from the fault and continue to receive power from local sources. So, the power continuity at Local Bus is improved. Comparing to [2] in which the controller only trips FCL_DG, the new DG still continually supplies power to local loads during and after faults by applying this dual-FCL connection. It is important to maintain the power continuity for some critical loads in local networks. At the same time, the accelerating torque acting on the prime mover of turbine-generator is decreased by keeping power supply to local loads, as shown in (1) and Fig. 1. Equation (1) demonstrates the synchronous equilibrium between the consumed power and produced power on the prime mover [3]. The accelerating energy with dual-FCL connection is less than the energy without this connection the electromagnetic torque is not zero so the acceleration of the DG's frequency is limited and the synchronism between the DG and the utility is enhanced [3].

$$
2 \mathrm{H} \frac{d w}{d t}=T_{m}-T_{e}
$$


Where $T_{m}$ is mechanical torque $T_{\varepsilon}$; is electromagnetic torque; $\mathrm{H}$ is the inertia constant of prime mover; is the rotating speed of shaft. FCLs work in a heavy limitation status (large X_DG and X_Grid) in this connection. Under a heavy limitation status, the fault current contribution of the DG is reduced to a low value, while more DGs can be added to the existing power grid without causing disturbances to the original protection relay scheme. After the faults are cleared, the cryogenic system drives HTS1 and HTS2 back into superconducting statuses. Both of Q1 and Q2 turn on to help the cooling of HTS wires by reducing jole heating. After HTS wires return to superconducting mode, the controller turns off Q1 and Q2. The main current route returns to HTS1 and HTS2, and it will not affect reclosing operation. The schematic diagram of the System is shown in the figure 6

\section{B. Control Scheme:}

The controller of the dual-FCL connection monitors current flows to distinguish the fault locations and trigger a proper FCL. The currents, which are detected by CS1 and CS2, are used as the inputs of the neuro fuzzy controller,

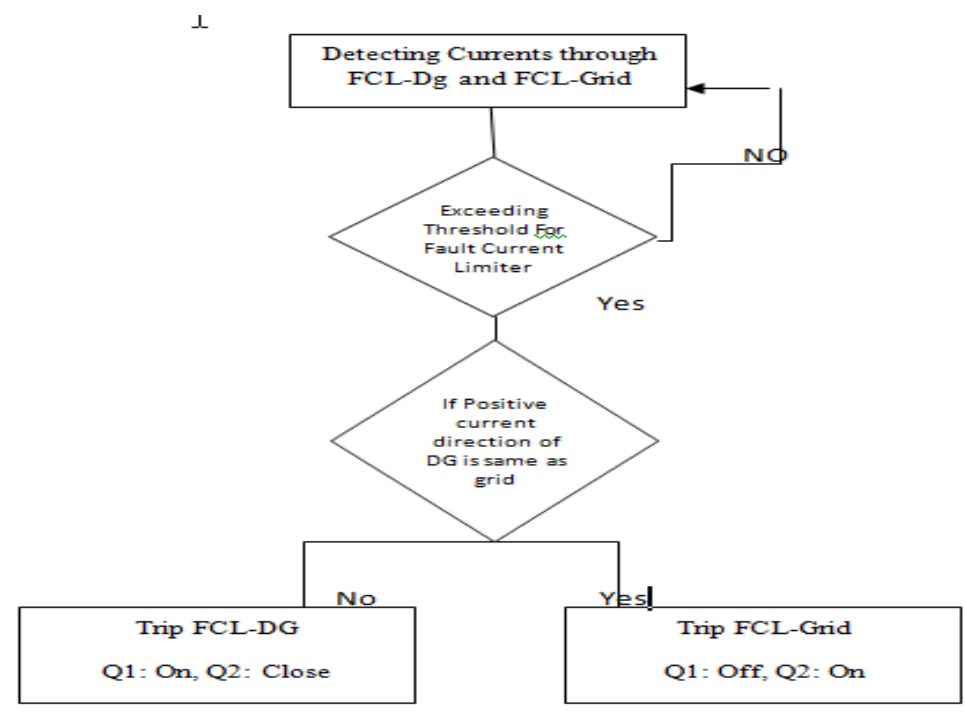

Fig. 7. Flow chart for the control scheme of dual-FCL connection.

Typically, the occurrence of a fault is distinguished when the fault current exceeds two times rated current of the DG with over-current relay scheme [4]-[6]. The positive direction of the current through FCL_DG is defined from the DG to Local Bus, while the positive direction of the current through FCL_Grid is defined from Local Bus to the Grid. FCL_DG and FCL_Grid locate close enough that the phase difference between their fault currents through are negligible during the fault occurring in the Grid. When the positive current direction of FCL_DG is the same as the positive current-direction FCL_Grid, the controller determines that the fault occurs at other places in the Grid other than Local Bus, and triggers FCL_Grid. Otherwise, the controller determines that the fault occurs at Local Bus and triggers FCL_DG.

\section{Simulation Results}

\section{A. Simulation Results When a Fault Occurs at Local Bus with Pi Controller:}

When a fault occurs at Local Bus, FCL DG is triggered, which is the same case as in [2]. The fault occurs at $90 \mathrm{~s}$ and is cleared in 5 cycles. When $\overline{F C}$ is not connected the fault increased from $47 \mathrm{Kv}$ to $91 \mathrm{KV}$. It can be observed that there is a large fault current contribution of $56 \mathrm{kA}$ from the DG into Local Bus. The excessive fault current flow may exceed the interrupting capacity ratings of $\mathrm{CBs}$, disturbs the original protection relay scheme, and prevents fault currents from being cut the fault current flowing into Local Bus is limited to 42 $\mathrm{kA}$. Only the fault current contribution of $1.0 \mathrm{kA}$ flows from the new DG. The fault current contribution can be further limited by increasing the maximum impedance of FCLs. In this way, the fault current is limited to less than the interrupting capacity ratings of the existing CBs. Fig 8 represent the active power from the system of Dg, Load and GRID. When the fault aloccurs in Dg and Grid there is a vast change in the Active power of too. 


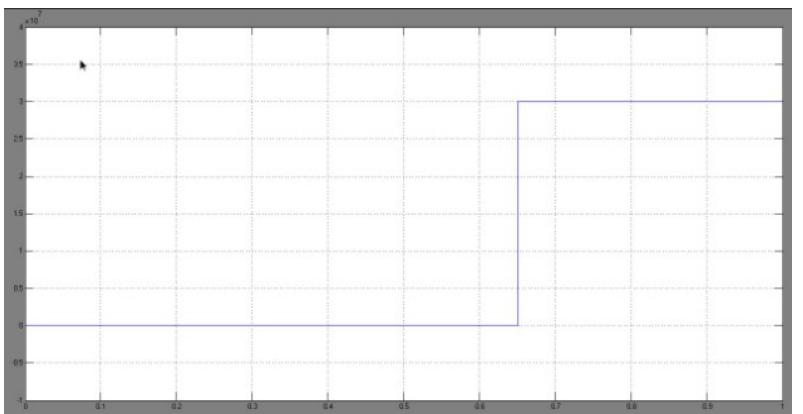

(A)

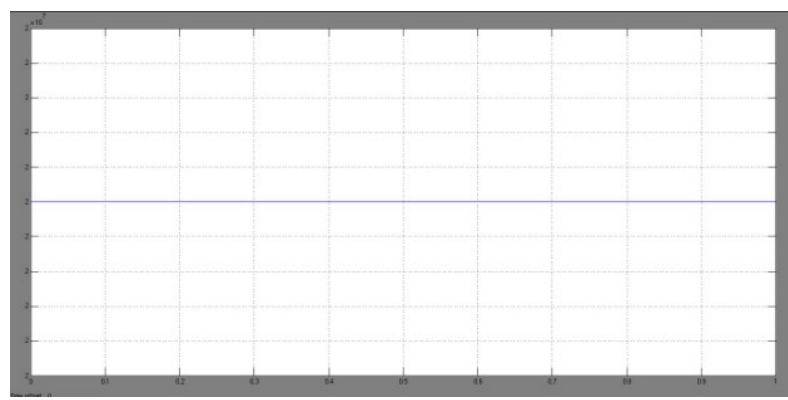

(B)

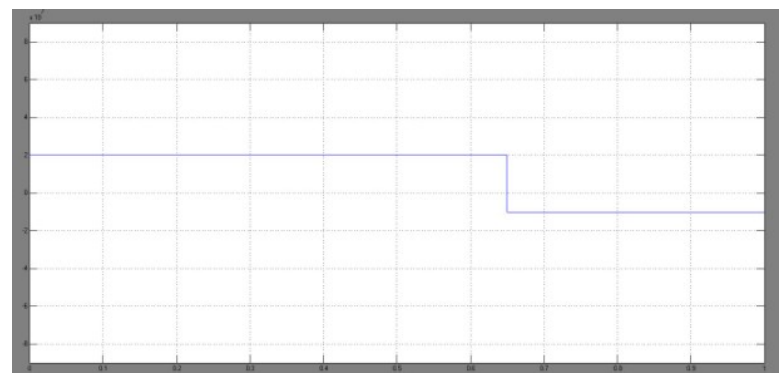

(C)

Fig 8: Active power from DG (A), LOAD (B) Grid(C)

Fig 9 indicates the currents flowing through when Dg is connected and no- fcl is installed and we can absard from the graph that the Dg is able to supply the power to the load (Local) .and Fig 10 represents the Current at local Bus with FCL and Fig 11 represents the currents without Dg and FCL and we see the improvement from the Graph.

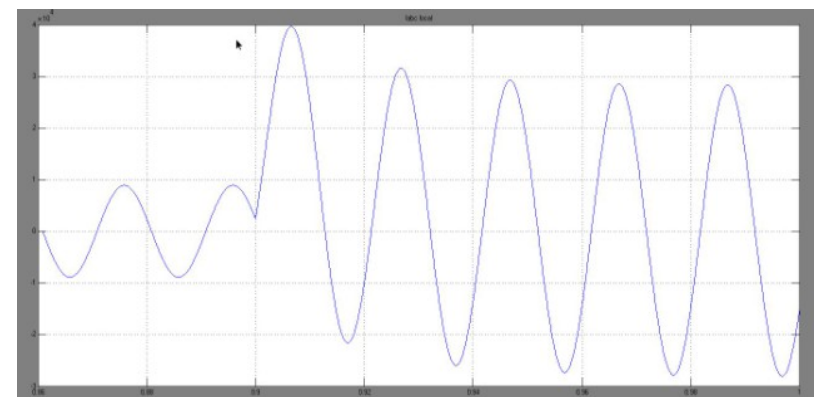

Fig 9: Current at Local Bus with DG No-FCL 


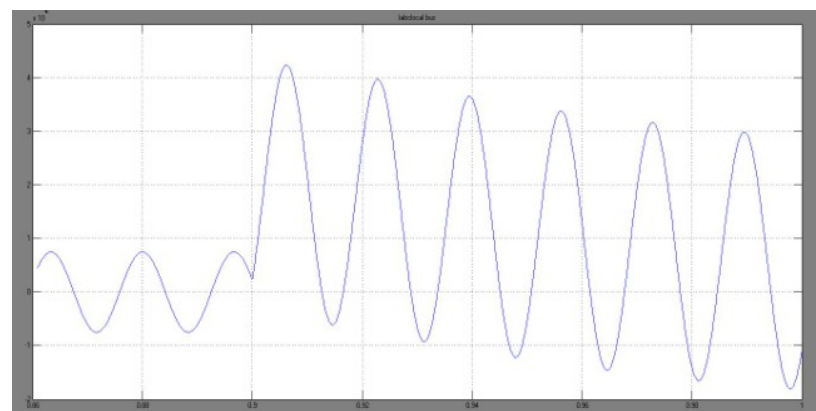

Fig 10: Currents at Local Bus with FCL

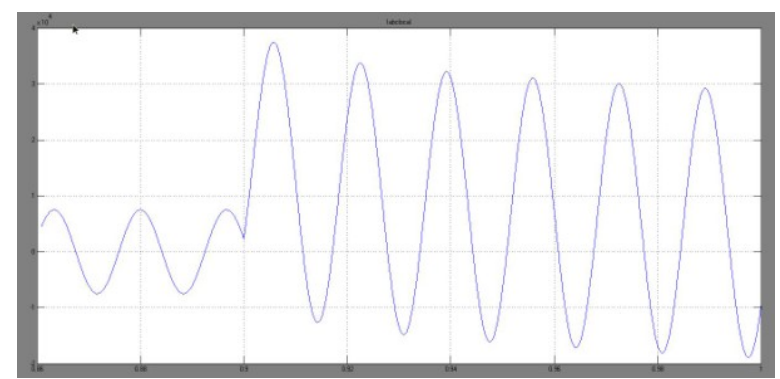

Fig 11: Without DG with FCL

\section{B. Simulation Results When a Fault Occurs at Local Bus with Neuro-Fuzzy Controller:}

Fig 12, 13 represents the local bus currents when $\mathrm{FCl}$ is installed and without respectively and as compared to fuzzy logic controller there is a high compensation level and $25 \%$ improvement has been observed.

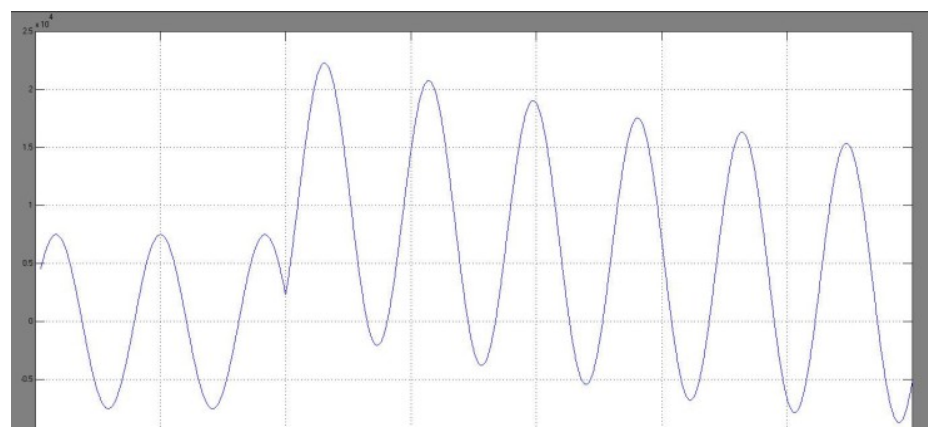

Fig 12: With FCL Iabc local

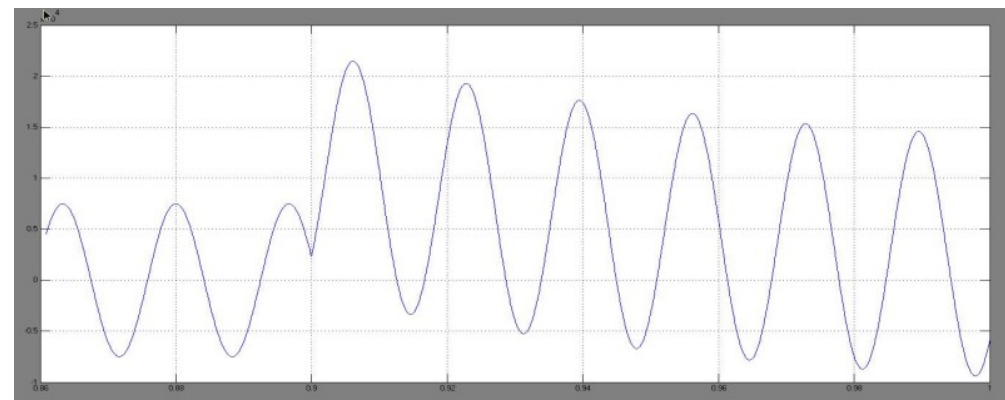

Fig 13: Without DG with FCL

\section{Simulations When a Fault Occurs in the Infinite System with Pi Controller:}

When a fault occurs at Fault_2, FCL_Grid is triggered. Fault_2 can represent all cases of fault conditions in the infinite power system, although the fault current contributions of the DG may be different depending on the line impedance between Local Bus and Fault_2. The fault occurs at $90 \mathrm{~s}$ and is cleared in 5 cycles. compares the fault current contribution of DG with and without the FCL, when a fault occurs in the infinite system. Without the FCL installed, the fault current flowing from the DG to the existing power grid is $4.8 \mathrm{kA}$. After the FCL is installed, the fault current contribution of the DG is limited to $0.4 \mathrm{kA}$ and does not disturb the original protection relay scheme in this case. With the increase of the maximum impedance of the 
FCL, the fault current can be limited to an even smaller value. The fault current contribution of the DG can be limited by tripping either FCL_DG or FCL_Grid. But, the benefits of tripping FCL_Grid, instead of FCL_DG, can be demonstrated by comparing the voltage stability and the frequency stability at Local Bus the voltage stability at Bus-i by tripping either FCL_DG or FCL_Grid. When FCL_DG is tripped, the voltage sag is 851 V, which corresponds to $25.33 \%$ of rated voltage. But, when FCL_Grid is tripped, the voltage sag is only $32 \mathrm{~V}$, which corresponds to $0.9 \%$ of rated voltage. Therefore, the voltage stability and power continuity at Local Bus is improved by tripping FCL_Grid, comparing to tripping FCL_DG. Moreover, the frequency oscillation of the DG can be limited by tripping FCL_Grid, instead of FCL_DG. In Fig. 10, when FCL_DG is tripped during a fault, the deviation of frequency reaches $0.14 \mathrm{~Hz}$, the same as without the FCL installed. The system returns to steady-state in $5 \mathrm{~s}$. But, when FCL_Grid is tripped, the deviation of frequency is limited to $0.01 \mathrm{~Hz}$ and the system returns to steady-state in less than 1.5 seconds. The speed-up of the DG is limited because the DG continues to supply the local loads at Local_Bus with FCL_Grid triggered during the fault.

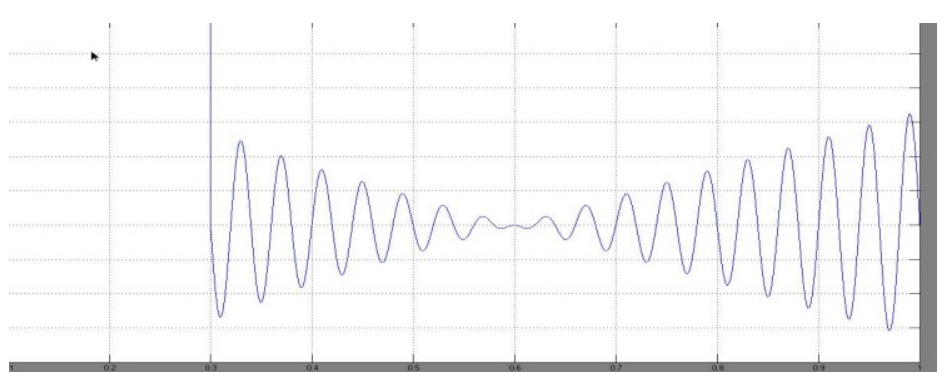

Fig 13: DG Frequency

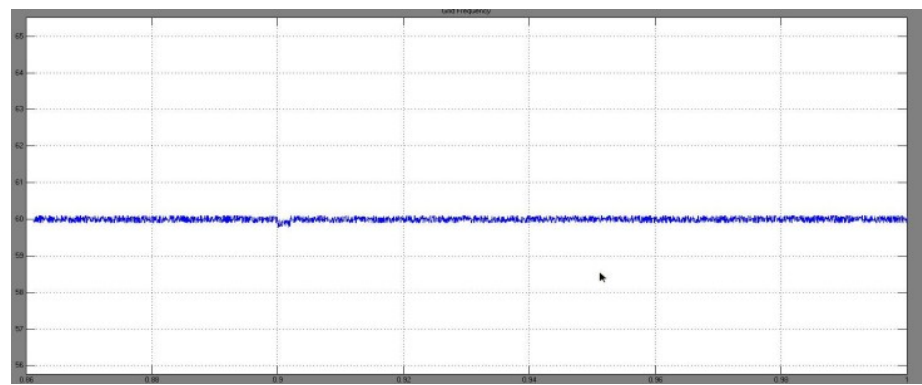

Fig 14: Grid Frequency

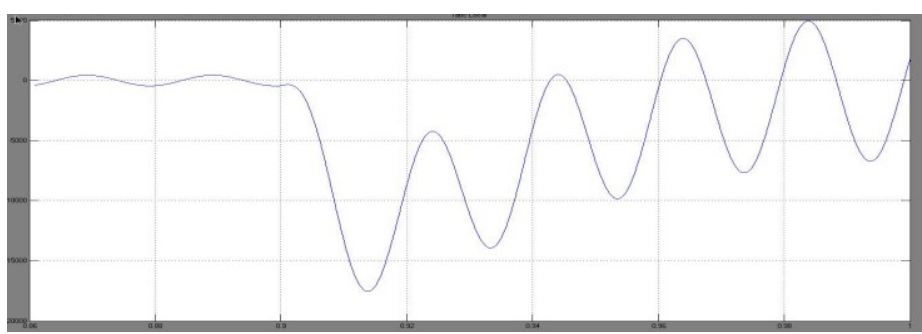

Fig 15: With DG no FCL

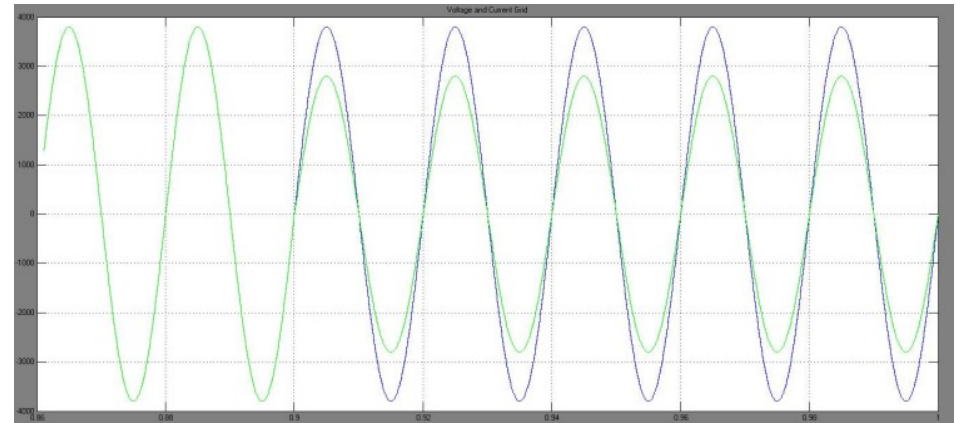

Fig 16: Vabc, Iabc 
Fig 13, 14 indicates the DG frequency and Grid frequency and fig 15 indicates the local bus currents with DG and no-FCl installed and fig16 represents the Voltage and currents across the Grid and we can absard that when the fault occurs at infinite bus there is a change in the phase of the grid Currents..

\section{Simulation results with ANFIS:}

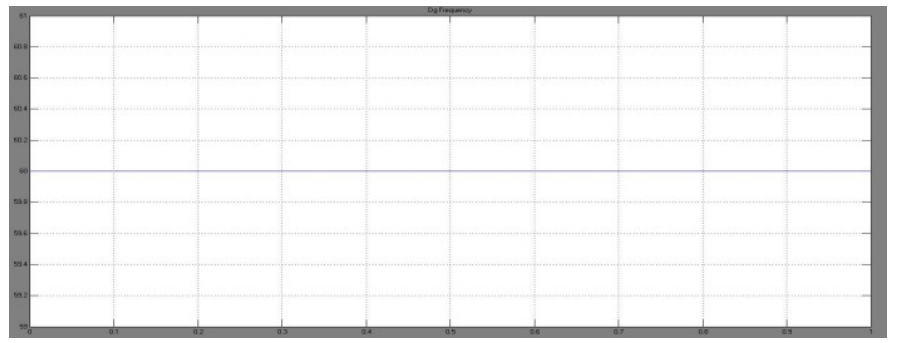

Fig 17: Frequency DG

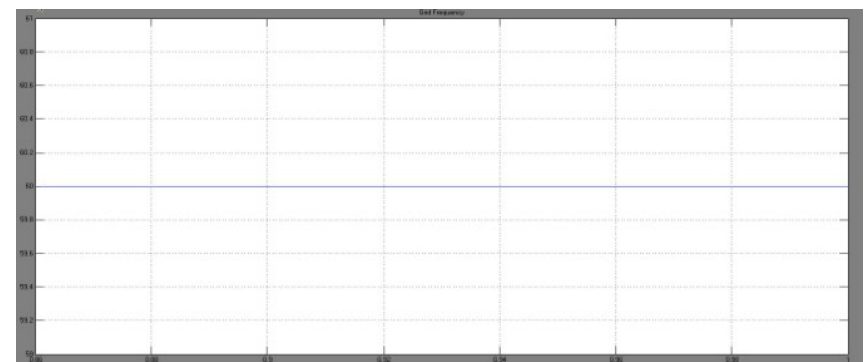

Fig 18: Grid Frequency

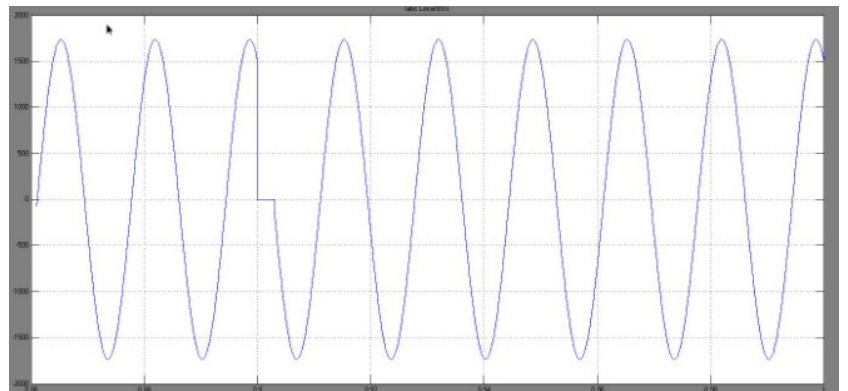

Fig 19: Iabc Local Bus

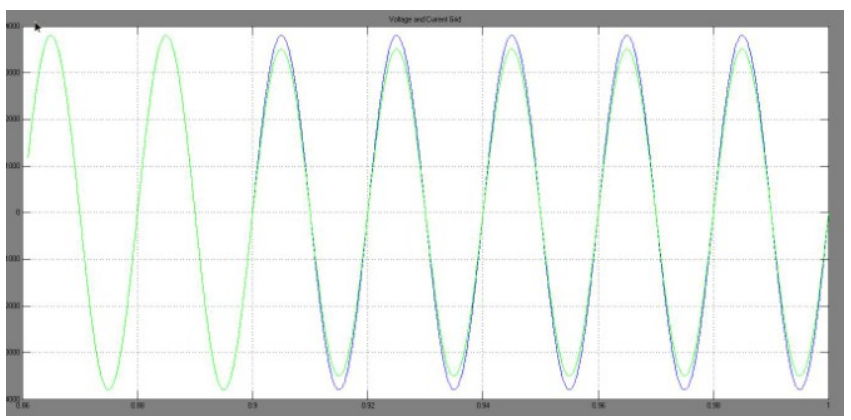

Fig 20: Vabc and Iabc Local

Fig 17,18 indicates the DG frequency and Grid frequency and fig 19indicates the local bus currents with DG and no-FCl installed and fig20 represents the Voltage and currents across the Grid and we can absorb that when the fault occurs at infinite bus there is a change in the phase of the grid Currents. And we can Cleary see that the neuro-fuzzy controller is able to control the FCL more accurately and the frequency is maintained constant and voltage and currents are in synchronous with each other.

\section{Conclusion}

A Neuro-fuzzy logic controller based novel dual-FCL connection is designed for connecting new DGs to the utilities. The fault current contributions of the DGs are limited, so it is not necessary to change the 
original protection relay scheme or to upgrade the existing CBs. Comparing to a single FCL device, this dualFCL connection costs only one more three-phase HTS wire and one more power switch to increase the synchronism between the new DGs and the utilities. Also, the power continuity at the bus connected to the new DG is enhanced because voltage sag is eliminated. It is indicates the Dual FCL is able to work more accurately with ANFIS controller compared with PI controller.. In future, the system can be further developed using Optimization technique or with ' $\mathrm{N}$ ' FCL Connection. The topology and control scheme of the dual-FCL connection are proposed, analysed and verified by simulation tests.

\section{References}

[1]. yucheng Zhang, and Roger A. Dougal "Novel Dual-FCL Connection for Adding Distributed Genration to a Power Distribution Utility” IEEE Trans. Appl. Supercond., vol. 21, no. 3,june 2011.

[2]. J. R. S. S. Kumara, A. Atputharajah, and J. B. Ekanayake et al., "Over current protection coordination of distribution networks with fault current limiters," in Proc. IEEE Power Eng. Soc. General Meeting, 2006, pp. 1-8.

[3]. J. D. Glover and M. S. Sarma, Power System Analysis and Design, $3^{\text {rd }}$ ed. San Anselmo, CA: Brooks/Cole, 2001.

[4]. V. Sokolovsky, V. Meerovich, and I. Vajda et al., "Superconducting FCL: Design and application," IEEE Trans. Appl. Supercond., vol. 14 , no. 3, pp. 1990-2000, Sep. 2004

[5]. IEEE Standard Rating Structure for AC High-Voltage Circuit Breakers Rated on a Symmetrical Current Basis, IEEE C37.04-1999.

[6]. IEEE Application Guide for AC High-Voltage Circuit Breakers Rated on a Symmetrical Current Basis, IEEE C37.010-1999.

[7]. T. Lovett, R. A. Dougal, A. Monti, and E. Santi, "A Multilanguage environment for interactive simulation and development of controls for power electronics," in Proc. IEEE Power Electron. Specialists Conf., Vancouver, Canada, 2001, vol. 3, pp. 1725-1729.

[8]. Y. Zhang, R. A. Dougal, and S. B. Kuznetsov, "Influence of inductive fault current limiter on generator synchronization," Naval Eng. J., to be published.

[9]. W. I. Rowen, "Simplified mathematical representations of heavy-duty gas turbines," ASME J. Eng. Power, vol. 105, pp. 865-869, 1983.

[10]. P. Kundur, Power System Stability and Control. New York: McGraw- Hill, 1993.

[11]. N. Idir, Y. Weens, and J.-J. Franchaud, "Skin effect and dielectric loss models of power cables," IEEE Trans. Dielectrics Electr. Insulation, vol. 16, no. 1, Feb. 2009.

[12]. Woodward, "Speed droop and power generation," application note 01302 [Online]. Available: http://www.canadiancontrols.com/documents/t technical/Speed\%20Droop\%20and\%20Power\%20Generation. Pdf

[13]. M. Andrus, S.Woodruff, and W. Ren, "Simulations of islanded generator synchronization in a notional integrated power system," [Online]. Available: http://esrdc.caps.fsu.edu/library.lookup.date.php

[14]. P. M. Anderson and A. A. Fouad, Power System Stability and Control, 2nd ed. Hoboken, NJ: Wiley-IEEE Press, 2002.

[15]. J. Driesen, P. Vermeyen, and R. Belmans, "Protection issues in microgrids with multiple distributed generation units," in Power Conversion Conf., Nagoya, April 2007, pp. 646-653.

[16]. W. Friedl, L. Fickert, E. Schmautzer, and C. Obkircher, "Safety and reliability for smart-, micro-, and islanded grids," presented at the CIRED Seminar: SmartGrids for Distribution, Jun. 2008, Paper 107.

[17]. L. Dessaint, K. Al-Haddad, H. Le-Huy, G. Sybille, and P. Brunelle, "A power system tool based on simulink," IEEE Trans. Industrial Electron., vol. 46, no. 6, pp. 1252-1254, Dec. 1999.

[18]. K. Maki, S. Repo, and P. Jarventausta, "Effect of wind power based distributed generation on protection of distribution network," in IEEE Developments in Power System Protection, Dec. 2004, vol. 1, pp. 327-330.

[19]. Simulation Study on A New Solid State Fault Current Limiter for High-Voltage Power Systems. Gang Chen, Daozhuo Jiang, Zhengyu Lu, and Zhaolin Wu, 2004 IEEE International Conference on Electric Utility deregulation, restructuring and Power Technologies (drpt2004) April 2004 Hong kong.

[20]. A New Proposal for Solid State Fault Current Limiter and Its Control Strategies. Gang Chen, Daozhuo Jiang, Zhengyu Lu, and Zhaolin $\mathrm{Wu}$

[21]. 3-Phase Fault Current Limiter for distribution systems Vijay K. Sood, Fellow, IEEE, and Shahabur Alam. 0-7803-9772X/06/\$20.00 2006 IEEE

[22]. V Yuvaraj and T Vasanth "Simulation, Control and Analysis of HTS Resistive and Power Electronic FCL for Fault Current Limitation and Voltage sag Mitigation in Electrical Network", IJEET vol.4, Issue 3, May -June(2013) PP. 82-94.

[23]. Neuro Fuzzy Systems:State-of-the-art Modeling Techniques. Ajith AbraHam

[24]. D. Glover and M. S. Sarma, Power System Analysis and Design, $3^{\text {rd }}$ ed. San Anselmo, CA: Brooks/Cole, 2001. 